\title{
Zukunftsgestaltung Waldorfschule
}


Steffen Koolmann

Joseph E. Nörling (Hrsg.)

\section{Zukunftsgestaltung Waldorfschule}

Ergebnisse einer empirischen Untersuchung zu Kultur, Management und Entwicklung

Mit einem Geleitwort von Prof. Dr. Horst Philipp Bauer Vorstand SOFTWARE AG - STIFTUNG

Springer VS 
Herausgeber

Steffen Koolmann

Institut für Bildungsökonomie

Alanus Hochschule für Kunst und Gesellschaft

Alfter, Deutschland
Joseph E. Nörling

Institut für Bildungsökonomie

Alanus Hochschule für Kunst und Gesellschaft

Alfter, Deutschland

ISBN 978-3-658-08983-2

ISBN 978-3-658-08984-9 (eBook)

DOI 10.1007/978-3-658-08984-9

Die Deutsche Nationalbibliothek verzeichnet diese Publikation in der Deutschen Nationalbibliografie; detaillierte bibliografische Daten sind im Internet über http://dnb.d-nb.de abrufbar.

\section{Springer VS}

(C) Springer Fachmedien Wiesbaden 2015

Das Werk einschließlich aller seiner Teile ist urheberrechtlich geschützt. Jede Verwertung, die nicht ausdrücklich vom Urheberrechtsgesetz zugelassen ist, bedarf der vorherigen Zustimmung des Verlags. Das gilt insbesondere für Vervielfältigungen, Bearbeitungen, Übersetzungen, Mikroverfilmungen und die Einspeicherung und Verarbeitung in elektronischen Systemen.

Die Wiedergabe von Gebrauchsnamen, Handelsnamen, Warenbezeichnungen usw. in diesem Werk berechtigt auch ohne besondere Kennzeichnung nicht zu der Annahme, dass solche Namen im Sinne der Warenzeichen- und Markenschutz-Gesetzgebung als frei zu betrachten wären und daher von jedermann benutzt werden dürften.

Der Verlag, die Autoren und die Herausgeber gehen davon aus, dass die Angaben und Informationen in diesem Werk zum Zeitpunkt der Veröffentlichung vollständig und korrekt sind. Weder der Verlag noch die Autoren oder die Herausgeber übernehmen, ausdrücklich oder implizit, Gewähr für den Inhalt des Werkes, etwaige Fehler oder Äußerungen.

Gedruckt auf säurefreiem und chlorfrei gebleichtem Papier

Springer Fachmedien Wiesbaden ist Teil der Fachverlagsgruppe Springer Science+Business Media (www.springer.com) 
Wir widmen dieses Buch Herrn Dr. BENEDIKTUS HARDORP, der uns stets als Förderer und interessierter Ratgeber des INSTITUTS FÜR BILDUNGSÖKONOMIE freundlichkritisch begleitete.

Alfter, im September 2014 


\section{Geleitwort}

Die Sicherung der Zukunftsfähigkeit von Schule und Unterricht ist in der heutigen Zeit eine wesentliche Herausforderung. Dies gilt umso mehr für die Schulen in freier Trägerschaft, die sich als Angebotsschulen gegenüber vielen konkurrierenden Einrichtungen - sei es in öffentlicher oder freier Trägerschaft - bewähren müssen. Darunter sind an prominenter Stelle auch die Freien Waldorfschulen zu subsumieren.

Konzeptionell betrachtet ist jede Freie Waldorfschule eine Schule mit Selbstverwaltung: Jedes hauptberuflich tätige Mitglied der Waldorfschulgemeinschaft muss seinen Beitrag dazu leisten, an der Verwaltung der Schule von seinem jeweiligen Platz aus mitzuwirken. An vorderster Stelle steht dabei der pädagogische Auftrag der Schule. Für die Lehrkräfte geht es primär um die Realisierung des pädagogischen Konzepts, unter anderem mittels des Systems der Lehrerkonferenzen.

Als Schulen in freier Trägerschaft müssen die Waldorfschulen aber auch mit unternehmerischem Geschick erfolgreich geführt werden. Um die wirtschaftlich-rechtlichen Belange kümmern sich die Schulträger, die meistens als Vereine konstituiert sind und die Durchführung des Schulbetriebs entweder als geschäftsführende Vorstände selbst wahrnehmen oder überwiegend auf angestellte Geschäftsführer übertragen. Gleichzeitig sollen auch die pädagogisch Tätigen in die wirtschaftlich-rechtlichen Belange der Selbstverwaltung eingebunden sein.

Hinzu kommt, dass in Deutschland die öffentliche Finanzierung für Schulen in freier Trägerschaft im internationalen Vergleich unterdurchschnittlich und unzureichend ist und Privatinitiativen eher behindert werden. Das Schulwesen ist heute vielerorts durch Bürokratisierung, Verrechtlichung, Überregulierung und unnötigen Staatseingriff gekennzeichnet. Durch diese Belastungen fehlt es häufig an der Bereitschaft zur Übernahme weiterer individueller Verantwortung durch den einzelnen Mitarbeiter über den pädagogischen Auftrag hinaus.

Gerade in Bezug auf die verschiedenen Herangehensweisen und Problemstellungen bei der wirtschaftlich-rechtlichen Führung einer Waldorfschule gibt 
es bislang kaum gesichertes Datenmaterial. Darin besteht der Verdienst dieser Studie, die sich dem Ansatz der AlanUS HOCHSCHULE verpflichtet fühlt, sämtliche Phänomene der "Waldorfwelt" einer empirischen Analyse zu unterziehen. Die Ergebnisse dieser Studie basieren auf der Auswertung einer Befragung von Geschäftsführern an deutschen Waldorfschulen. Als Folgepublikation zu dem bereits von DIRK RANDOLL herausgegebenen Band "Ich bin Waldorflehrer", der eine umfangreiche Betrachtung der Situation der Waldorflehrer beinhaltet, schließt diese Untersuchung eine Lücke, die über die Deskription des pädagogischen Geschehens der Waldorfschulen hinausgeht und die Waldorfschulen auch als wirtschaftlich agierende Organisationen versteht.

Die Studie unterzieht die Zukunftsfähigkeit der Waldorfschulen anhand der drei Schwerpunkte „Organisationskultur und Werte", „Führung und Management" sowie "Lernen und Organisationsentwicklung" einer kritischen Analyse und nimmt dabei vor allem die Perspektive der in der Geschäftsführung tätigen Mitarbeiter in den Blick, die letztlich die Verantwortung für den wirtschaftlichen Erfolg der Schulen tragen. Die Autoren verknüpfen dabei die empirischen Ergebnisse ihrer Erhebung mit aktuellen Ansätzen aus der Betriebswirtschaftslehre zu erfolgreicher Unternehmensführung. Die untersuchten Waldorfschulen wurden aus einer organisationstheoretischen Perspektive betrachtet, mit dem Ziel, Schwachstellen in der gegenwärtigen Selbstverwaltung aufzuzeigen und Hinweise für eine zukunftsfähige und erfolgreiche Ausgestaltung der Schulverwaltungsarbeit zu geben.

Dass es bezüglich der organisatorischen Belange Handlungsbedarf gibt, dürfte jedem klar sein, der sich näher mit dem „Phänomen Waldorfschule“ beschäftigt hat. In ihren Untersuchungen gehen die Autoren dabei behutsam mit der Gegebenheit um, dass jede Schule individuelle Rückschlüsse aus den hier präsentierten Ergebnissen ziehen muss und die gewonnenen Erkenntnisse nicht pauschalisiert werden können. In jedem Fall lassen sich aus den Ergebnissen dieser Studie aber Tendenzen ableiten, die zu einer erfolgreichen Gestaltung der organisatorischen Zukunft der Waldorfschulen beitragen können. Ich wünsche dieser praxisorientierten Publikation eine gute Resonanz in den Schulen und der interessierten Fachöffentlichkeit.

Darmstadt, im September 2014

Prof. Dr. Horst Philipp Bauer Vorstand SOFTWARE AG - STIFTUNG 


\section{Danksagung}

Ein Werk, und sei es eine solche Forschungsarbeit, bedarf oftmals vieler Beteiligter, damit es im Nachgang zur ersten Idee tatsächlich zustande kommt. Das war in diesem Fall nicht anders.

Da war zunächst Professor Dr. DIRK RANDOLL, der es uns ermöglichte, dass wir uns mit einem Mantelfragebogen an die von ihm durchgeführte Lehrerstudie an Waldorfschulen anschließen konnten, und der uns im späteren Verlauf finanzielle Mittel für die Erarbeitung dieser Studie zur Verfügung stellte. Der DAMUS DONATA E. V. sorgte für die Finanzierung der wissenschaftlichen Mitarbeiterstelle und ermöglichte damit insbesondere die sorgsame und zeitintensive statistische Untersuchung des Datenmaterials. FLORIAN BOUKAL, der an der Mitgestaltung des Erhebungsdesigns beteiligt war, hat zudem die Verfassung eines ganzen Kapitels übernommen. Während der Auswertung war für uns immer wieder Dr. JÜRGEN PETERS ein wichtiger Rat- und Hinweisgeber. Herr Professor Dr. HORST PHILIPP BAUER, Geschäftsführender Vorstand der SOFTWARE AG-STIFTUNG, übernahm die Formulierung eines Geleitwortes. All diesen Menschen danken wir sehr herzlich; ohne ihre Unterstützung wäre diese Arbeit nicht entstanden.

Dennoch: All die Bemühungen dieser Beteiligten - inklusive unserer eigenen als Verantwortliche und Durchführende - wären allein nicht ausreichend gewesen, um diese Forschungsarbeit zu erstellen. Denn ohne die Mitarbeit der Geschäftsführerinnen und Geschäftsführer der Waldorfschulen hätte uns schlichtweg das Material gefehlt, das es nun einmal für wissenschaftliche Forschung braucht. Insofern: Recht herzlichen Dank an all diejenigen, die es nicht gescheut haben, sich zusätzlich zu ihren umfangreichen Aufgaben in den Waldorfschulen die Zeit zu nehmen, unseren Fragebogen auszufüllen. Sie, liebe Geschäftsführerinnen und Geschäftsführer, sehen bzw. lesen nun, was dabei heraus gekommen ist - und mögen selbst entscheiden, ob sich Ihr Engagement gelohnt hat.

Alfter, im September 2014

Prof. Dr. Steffen Koolmann und Joseph E. Nörling INSTITUT FÜR BILDUNGSÖKONOMIE 


\section{Inhaltsverzeichnis}

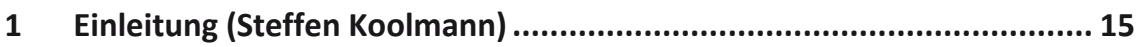

2 Konzeptionelle Gestaltung der Studie

(Steffen Koolmann/Joseph E. Nörling) ................................................ 19

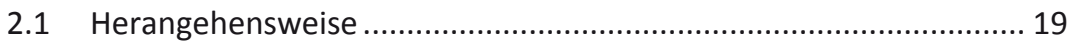

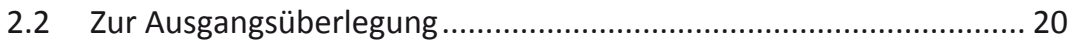

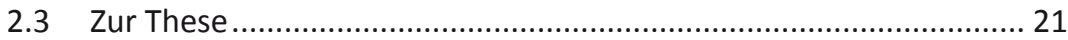

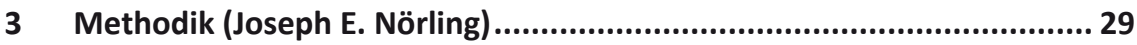

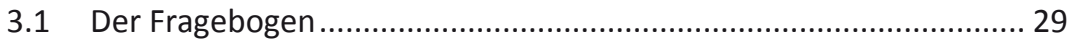

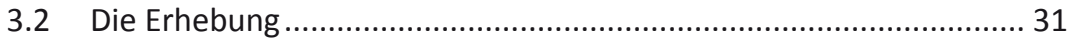

4 Merkmale der Stichprobe (Joseph E. Nörling)..................................... 33

4.1 Schulische Rahmenbedingungen...................................................... 33

4.1.1 Geschichtliche Hintergründe der

Waldorfschulbewegung, Schulalter und Gemeindegröße..... 33

4.1.2 Schüler und Lehrer .............................................................. 39

4.1.3 Ausbaugrad, Anzahl der Züge, Klassengröße........................ 44

4.2 Gebäude und Raumsituation sowie Bautätigkeit.............................. 52

4.2.1 Schulgebäude und Investitionen in Neubauten .................... 52

4.2.2 Größe der Räumlichkeiten.................................................. 57

4.2.3 Qualität und Ausstattung der Räumlichkeiten ...................... 60

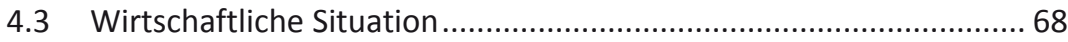

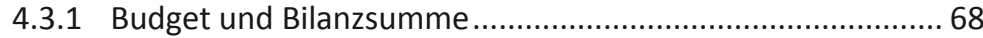

4.3.2 Jahresergebnis und Verbindlichkeiten .............................. 73

$5 \quad$ Kultur und Werte (Joseph E. Nörling) .................................................... 81

5.1 Grundlagen der Organisationskultur ............................................... 81

5.2 Das Verhältnis der Waldorfschulen zum örtlichen Umfeld ............... 87 
5.2.1 Öffentlicher Ruf der Schule und Kooperationen mit

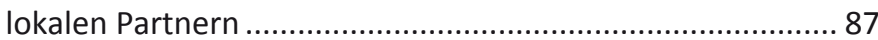

5.2.2 Die Sozialstruktur der Elternschaft im Umfeld der Schule .... 90

5.3 Der Umgang mit Streitigkeiten und Konflikten ............................... 92

5.3.1 Rechtsstreitigkeiten mit Lehrern und Eltern ........................ 92

5.3.2 Streit und Konfliktkultur .................................................... 93

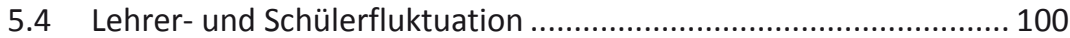

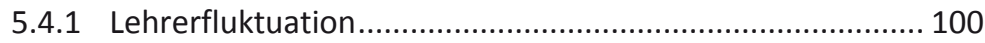

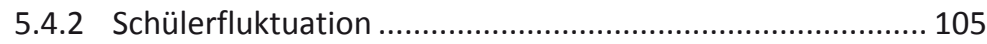

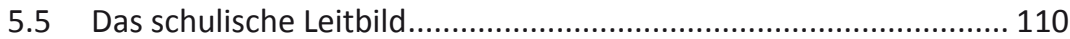

5.5.1 Bedeutung und Funktion des schulischen Leitbildes........... 110

5.5.2 Regelmäßige Leitbildarbeit............................................. 112

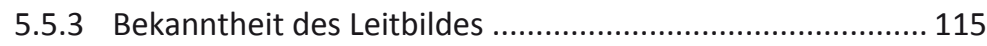

5.6 Die innere Überzeugung der Mitarbeiter...................................... 119

5.7 Gehaltsordnungen und betriebliche Altersversorgung ................... 126

5.7.1 Die Gehaltssysteme an Waldorfschulen .............................. 126

5.7.2 Das von den Geschäftsführern favorisierte

5.7.3 Die betriebliche Altersversorgung ....................................... 134

5.7.4 Umfang der Lehrerdeputate und Erfassung der Arbeitszeit........................................................................ 135

6 Führung und Management (Steffen Koolmann) ................................. 141

6.1 Führung und Management in Not-for-Profit-Organisationen.......... 141

6.2 Die schulische Selbstverwaltung ................................................ 143

6.2.1 Grundlagen und Herausforderungen der

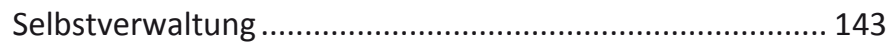

6.2.2 Zufriedenheit mit der Selbstverwaltungsarbeit .................. 146

6.2.3 Verpflichtung zur Mitarbeit bei Selbstverwaltung und Pädagogik ......................................................................... 150

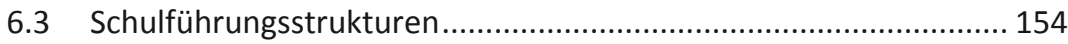

6.3.1 Die Formen der Schulführung an Waldorfschulen .............. 154

6.3.2 Verteilung der Arbeitsbelastung in der Schulführung ......... 159

6.4 Managementstrukturen .............................................................. 165 
6.4.1 Anstellungsverhältnis und Ausbildungshintergründe der

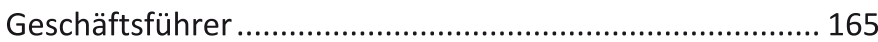

6.4.2 Eingerichtete Verwaltungsstellen.................................... 171

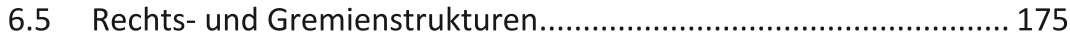

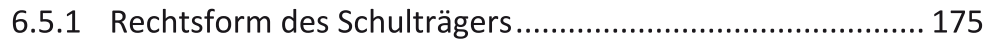

6.5.2 Eltern und Lehrer im Vorstand des Schulträgers................. 178

6.5.3 Eingerichtete Gremien und Corporate Governance............ 180

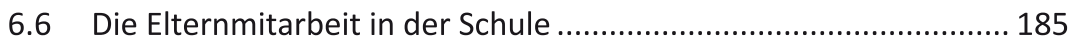

$7 \quad$ Lernen und Entwicklung (Florian Boukal) ........................................... 193

7.1 Lernen und Entwicklung als Aspekte der Zukunftsfähigkeit von

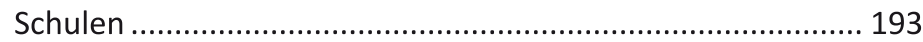

7.2 Maßnahmen zur Organisationsentwicklung ................................ 198

7.2.1 Interne Beschäftigung mit der Organisationsentwicklung .. 198

7.2.2 Externe Beratungen für den organisatorischen Bereich ..... 204

7.3 Mitarbeiter- und Lehrkräfteentwicklung....................................... 208

7.3.1 Die Bedeutung der Lehrkräfte für die Schulentwicklung..... 208

7.3.2 Nicht besetzte Lehrerstellen und deren Ursachen .............. 210

7.3.3 Motivation und Alter der Lehrkräfte ................................. 213

7.3.4 Personalentwicklung ..................................................... 217

7.4 Weiterentwicklung des Schulkonzepts ........................................ 225

7.4.1 Pädagogische Zusatzangebote ......................................... 225

7.4.2 Offenheit für externe Einflüsse in Pädagogik und Schulführung

8 Zukunftsherausforderungen aus Sicht der Geschäftsführer

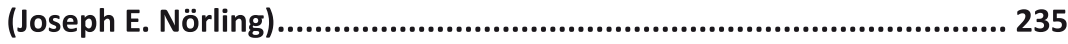

9 Fazit (Steffen Koolmann/Joseph E. Nörling) ................................... 245

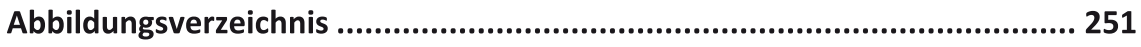

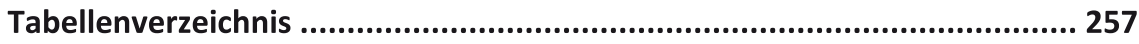

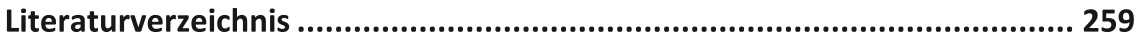

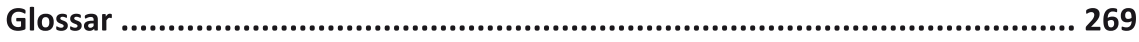

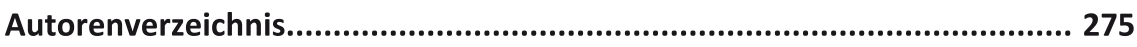

